\title{
Critical elasticity of polyacrylamide above its gel point
}

\author{
B. Gauthier-Manuel (*) and E. Guyon (**) \\ L.H.M.P., E.S.P.C.I., 10, rue Vauquelin, 75005 Paris, France
}

(Reçu le 15 juillet 1980, accepté le 5 septembre 1980)

\begin{abstract}
Résumé. - Nous avons étudié la polymérisation réticulaire d'un gel de mono et bisacrylamide autour de son seuil de gélation. Une mesure utilisant le principe du déplacement d'une bille de Stokes permet de caractériser le point de gel à partir de la divergence de la viscosité du temps $T=T_{\mathrm{c}}$ (évalué à partir du début de la polymérisation) et son élasticité de cisaillement $E(T)$ au-dessus de ce seuil. Une loi de comportement critique $E(T) \propto\left(T-T_{\mathrm{c}}\right)^{t}$ est obtenue pour : $2 \times 10^{-3}<\left(T-T_{\mathrm{c}}\right) / T_{\mathrm{c}}<10^{-1}$ avec une valeur de $t$ variant entre $1,9 \pm 0,2$ à $2,2 \pm 0,2$ suivant les conditions chimiques. Ce résultat est comparé aux prédictions des modèles de percolation.
\end{abstract}

\begin{abstract}
We study the reticular polymerization of a gel of mono and bisacrylamide around the gelation threshold. A rheological measurement using the Stokes relative displacement of a sphere within the medium gives access to the viscosity below the gel point and to an elastic modulus $E$ above it. From the first determination we deduce the gel time $T=T_{\mathrm{c}}$ (evaluated from the start of the polymerization). A critical variation $E(T) \propto\left(T-T_{\mathrm{c}}\right)^{t}$ is obtained for $2 \times 10^{-3}<\left(T-T_{\mathrm{c}}\right) / T_{\mathrm{c}}<10^{-1}$ with an exponent $t$ varying from $1.9 \pm 0.2$ to $2.2 \pm 0.2$ for different chemical conditions. The result is compared with percolation models of gelation.
\end{abstract}

The formation of an elastic gel above a critical amount $p=p_{\mathrm{c}}$ random branching of polymeric chains is an example of a percolation problem. The approach of Flory, Stockmayer [1] and Gordon [2] uses a Cayley tree model (without internal cyclization). On the other hand that of De Gennes [3] and Stauffer [4] emphasizes the role of loops and excluded volume not considered in a tree description. In principle the study of the rheological behaviour around $p_{\mathrm{c}}$ permits to choose between the two models. Below $p_{c}$, Adam et al. [5] observe a divergence of the viscosity of the sol phase of a chemical gel with a critical exponent close to that predicted by De Gennes. In this letter we describe, using the apparatus initially conceived by Pieranski [6], the elasticity of the gel above $p_{\mathrm{c}}$. In the critical range $0 \leqslant\left(p-p_{\mathrm{c}}\right) / p_{\mathrm{c}} \ll 1$, the shear elasticity modulus [7] is given by :

$$
E \sim\left(\left(p-p_{\mathrm{c}}\right) / p_{\mathrm{c}}\right)^{t} .
$$

We have used the copolymerization of a mixture of mono and bisacrylamide in aqueous solution $(1.5 \%$

(*) In partial fulfilment of a « thèse de $3^{\mathrm{e}}$ cycle ".

(**) And : Université de Paris Sud, 91405 Orsay, France. per weight) [8] under the influence of an initiator. The percentage of the «bis" monomer $(3 \%$ of the " mono") controls the rate of branching between linear chains (formed by homopolymerization of the monoacrylamide). The branching develops regularly as a function of the time variable $T$. As there is no reason to expect that the reactivity will be modified critically around $p_{\mathrm{c}}$, we replace the variable $p$ by $T$ which is legitimate for the study of critical behaviour in a range narrow enough around $p_{\mathrm{c}}$ (or $T_{\mathrm{c}}$ ).

The critical time has been defined in the following way : for smaller times, evaluated from the start of the polymerization, we follow the variation of viscosity $\eta$ by imposing a sawtooth displacement to the solution. The increase of $\eta(T)$ (Fig. 1) is felt only in the immediate vicinity of $T_{\mathrm{c}}$ (several minutes for a value of $T_{\mathrm{c}}$ of $16 \mathrm{~h}$ ) where divergence of $\eta$ is observed. The value of $T_{c}$ is estimated (Table I, column 3) from a linear extrapolation of $\eta(T)^{-1}$ to zero. It is not possible to characterize more precisely the critical behaviour below $T_{\mathrm{c}}$ as some viscoelastic effects are obtained very near $T_{\mathrm{c}}$ (for $\left|\Delta T / T_{\mathrm{c}}\right|<3 \times 10^{-3}$ ) which should be considered in a finite frequency analysis. This behaviour is qualitatively similar to that observed with polyox test solutions. The Maxwell 


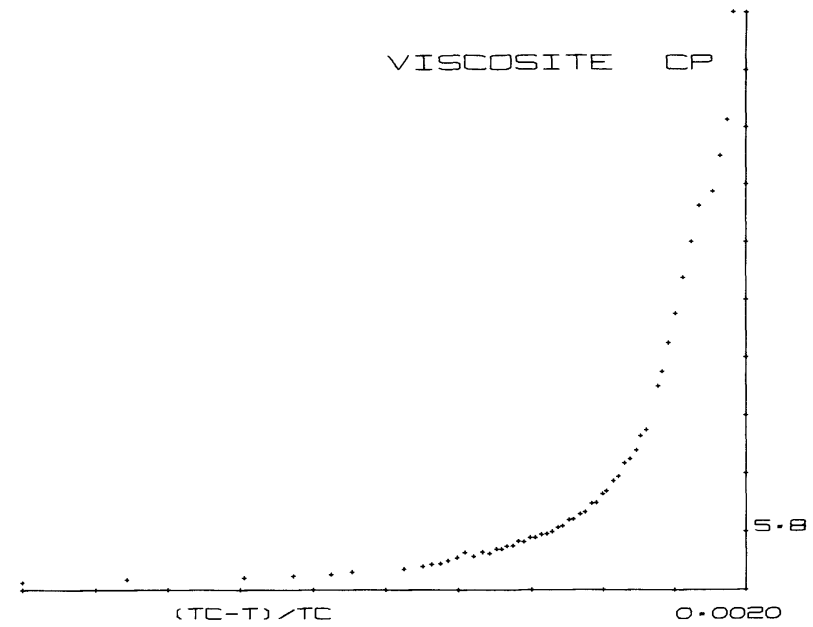

Fig. 1. - Experimental variation of the viscosity below the gel point in reduced units $\left(T_{\mathrm{c}}-T\right) / T_{\mathrm{c}}$. The intercept of the axis is the origin for both ordinates.

time constant increases very rapidly, as $T$ approaches $T_{\mathrm{c}}$, up to several seconds (which correspond to the period of the alternating motion of the cell).

The value of $T_{\mathrm{c}}$ is a function of the concentration of bisacrylamide, of the dilution of the solution, of the activator and of the temperature (kept to $31^{\circ}$ ). The reproducibility of independent experiments, keeping these parameters constant, is characterized by a variation of $T_{\mathrm{c}}$ less than $20 \mathrm{~min}$. over $16 \mathrm{~h}$, i.e. less than $2 \%$ !; after shifting the curves for the displacement of $T_{\mathrm{c}}$, the variations of $E(T)$ in different runs are perfectly reproducible within the limit of accuracy of the experiment $(1 \%)$.

The curve $t=1$ in figure 2 is the experimental variation of $E(T)$ for a typical gel. It shows the progressive increase of elasticity above $T_{c}$ already obtained qualitatively in the experiments of Peniche Covas et al. [9] on gelatin. The critical behaviour (form. (1)) is studied in figure 2 from a linear regression of $E^{1 / t}(T)$ for different values of $t$. When the exponent $t$ varies, the coefficient of the regression does not show important variations under the influence of the overall curvature when $t$ has not the optimal value. On the other hand the parameters of the regression (slope and extrapolated value $T_{\mathrm{c}}^{\prime}$ such that $\left.E^{1 / t}\left(T_{\mathrm{c}}^{\prime}\right)=0\right)$ vary much with the choice of $t$. It is the adjustment of the value of $T_{c}^{\prime}$ (line 2 in the table) with $T_{\mathrm{c}}$ which gives the most accurate deter-

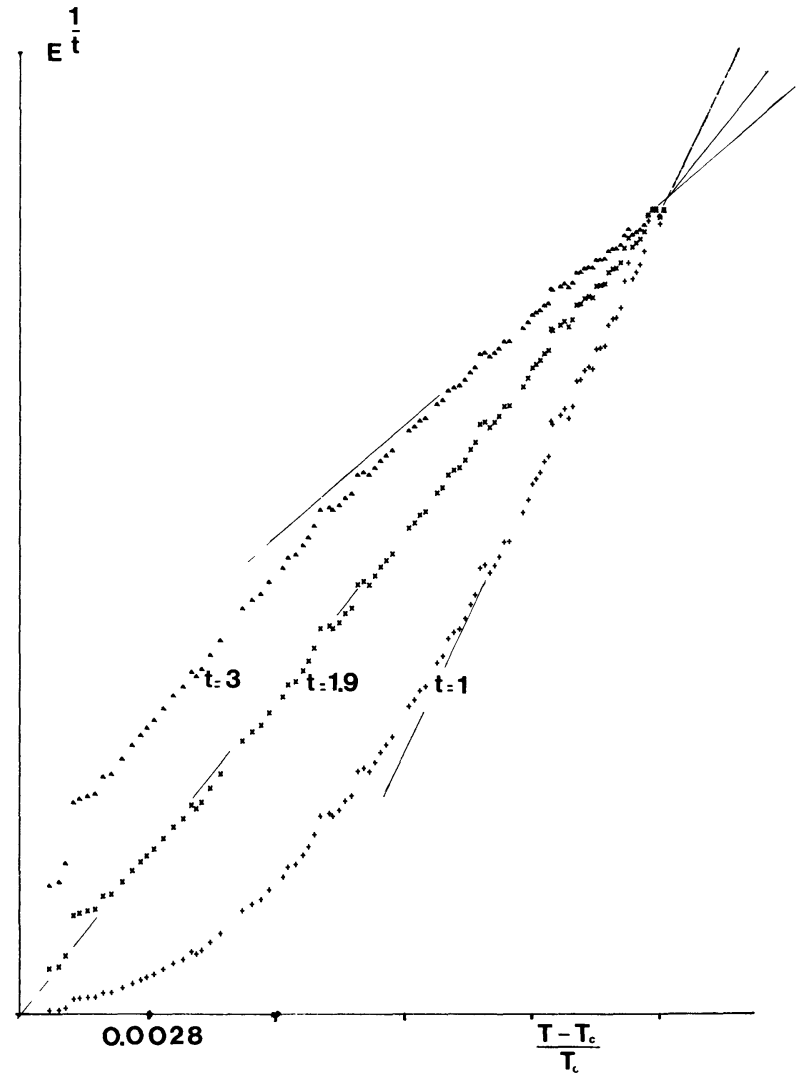

Fig. 2. - Experimental variation of the elastic modulus above the gel point in reduced units $\left(T-T_{\mathrm{c}}\right) / T_{\mathrm{c}}$ (curve $t=1$ ). The exponent $t=1.9$ gives the best linear variation of $E^{1 / t}$ with $T$, compatible with the value of $T_{c}$ deduced from the divergence of the viscosity.

mination of $t$. In the case of the sample of figure 2 we get $t=1.9 \pm 0.1$ in the interval

$$
0<\Delta T / T_{\mathrm{c}}<1.5 \times 10^{-2}
$$

with a correlation coefficient of 0.9993 .

Note. - In order to verify that the parameters (slope and $T_{\mathrm{c}}^{\prime}$ ) apply through this critical range we got a first determination using the first 40 data points and then following ones by adding points by groups of 10 . The variation of $T_{c}^{\prime}$ is smaller than $2 \times 10^{-4}$ and the slope remains constant within $5 \times 10^{-3}$.

In the present state of our experiments on this gel system (20 samples) the value of $t$ appears to depend slightly on the concentration of " bis " $(t$ reaches a

Table I.

Critical exponent $t$

$T_{\mathrm{c}}^{\prime}$; regression

$T_{\mathrm{c}}$; divergence of $\eta$

\section{$\begin{array}{ll}1.6 & 1.7\end{array}$}

$61148 \mathrm{~s} \quad 61125 \mathrm{~s}$
1.8

$61103 \mathrm{~s}$

$61078 \pm 6 \mathrm{~s}\left(^{*}\right)$
2.0
$61080 \mathrm{~s} \quad 61058 \mathrm{~s} \quad 61034 \mathrm{~s}$

2.1

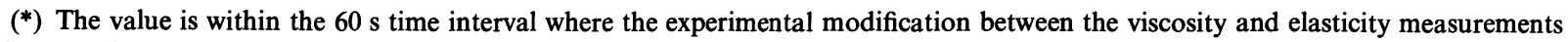
has been made. 
value $2.2 \pm 0.2$ if one increases the overall concentration of monomers by a factor 2).

Within this range of values one can compare the present results with theory. The percolation model of De Gennes and Stauffer uses an analogy between $E(p)$ and the electrical conductivity of a random network of conductiving (percentage $p$ ) and insulating $(1-p)$ bonds. A theoretical analysis of the latter problem, based on the notion of the formation of a superlattice above $p_{\mathrm{c}}$, leads to the relation

$$
t=v(d-2)+1
$$

where $d(=3)$ is the dimension of space and $v(0.8$ for $d=3$ ) is the critical exponent of the correlation length (the average length between two nodes of the superlattice). Electrical conductivity measurements on regular or random lattices give $t=1.5$ to 1.8 [10] which is not very far from the estimate (form. (2)). On the other hand, the treatment by Gordon using the tree approximation gives $t=3$, which is the value obtained in (2) for $d=6$ (the dimension above which critical effects - loops and excluded volume are negligible near percolation threshold).
Our experimental values are closer to that in the percolation limit, but we feel that the larger values we obtain are a significant disagreement.

The correlation effects introduced by the chemical process of formation of the gel (growth aspect of the process plus steric hindrance) can lead to deviations from the standard percolation results. This point was analysed recently in a numerical analysis by Manneville and de Seze [11]. Using a determination of the correlation length on finite size networks, where additive polymerization was simulated, and the relation (2), they also obtain values of $t$ larger than the percolation result. We hope to clarify the origin of the discrepancy, using the systematic variation of the chemical parameters.

Acknowledgments. - Antoinette Fleisch, in the course of her " stage de DEA de Physique des Solides ", contributed to the numerical analysis of the results. We benefited by several discussions with R. Audebert, P. G. De Gennes and E. Stanley and by the initial effort of R. B. Meyer and P. Piéranski who obtained the first results on this system.

\section{References}

[1] See for ex. : FLoRy, P., Principles of polymer chemistry (Cornell Univ. Press, Ithaca) 1953, chap. 9.

[2] Gordon, H., RG. CP Plastiques 4111 (1967).

[3] De Gennes, P. G., Scaling concept in polymer physics (Cornell Univ. Press, Ithaca) 1980.

[4] Stauffer, D., J. Chem. Soc. Faraday Trans. 72 (1976) 1354.

[5] Adam, M., Delsanti, M., Oxasha, R. and Wild, G., J. Physique Lett. 40 (1979) L-359.

[6] See also the results of : Gauthier-Manuel, B. and PieRANSKI, P., Patent in progress ;

GaUthier-Manuel, B., " stage de DEA » (1979). The value of the shear elastic modulus is proportional to the force exerted on a sphere located within the gel which undergoes a relative displacement with respect to the sphere. In the sol phase, one measures the force when the liquid is displaced at a relative constant velocity. The calibration on the two types of measurements is done by measuring the force exerted on a liquid of known viscosity.

[7] We measure the shear modulus from the Stokes force. However it is expected that the two elastic moduli have the same critical behaviour.

[8] See for ex. : Hochberg, A., Tanaka, T. and Nicoli, D., Phys. Rev. Lett. 43 (1979) 217.

[9] Peniche Covas, C., Dev, S., Gordon, M., Judd, M., KajIVARA, K., Gels and gelling processes, Faraday discuss. 57 (1974) 165.

[10] KirkPatrick, S., Rev. Mod. Phys. 45 (1973) 574.

[11] Manneville, P. and De Seze, L., To appear in Springer Series in Synergetics : Colloque sur les méthodes de calcul pour l'étude des phénomènes critiques, Carry (juin 1980). 